\title{
Assessing Barriers to Insulin Therapy among Omani Diabetic Patients Attending Three Main Diabetes Clinics in Muscat, Oman
}

*Abdulla Al Futaisi, ${ }^{1}$ Magdi Alosali, ${ }^{2}$ Ali Al-Kazrooni, ${ }^{3}$ Salim Al-Qassabi, ${ }^{2}$ Sumaia Al-Gharabi, ${ }^{4}$ Sathiya Panchatcharam, ${ }^{5}$ Abdulaziz M. Al-Mahrezi ${ }^{6}$

\begin{abstract}
Objectives: This study aimed to identify the main barriers that prevent patients with diabetes mellitus from accepting insulin therapy. Methods: This cross-sectional study was conducted from May to December 2019. Convenience sampling was used to recruit participants from three diabetes clinics in Muscat, Oman. Eligible participants were interviewed in person based on a pre-prepared questionnaire. The questionnaire, which was administered in Arabic, includes demographic data and 19 specific items on barriers to insulin therapy. Results: A total of 201 participants (response rate: 93\%) were enrolled in the study. The most common barriers were as follows: concern of frequent blood glucose checking (36.3\%), long-term injections (33.8\%), side-effects of insulin (29.9\%) and weight gain (29.4\%). Needle phobia was considered a barrier by only 9\% of the participants. Overall, 125 (62.2\%) participants were willing to initiate insulin therapy despite the presence of these barriers and only 20 (10\%) of them were influenced by these barriers to such a degree that they rejected the insulin therapy. Conclusion: The majority of participants had no identifiable reasons to stop them from accepting insulin therapy. Effective strategies should be developed to address each of the main barriers to improve acceptance and adherence to insulin therapy.
\end{abstract}

Keywords: Diabetes Mellitus; Insulin; Treatment Refusal; Needles; Phobic Disorders; Hypoglycemia; Weight Gain; Oman.

\section{ADVANCES IN KNOWLEDGE}

This study found that the main barriers to the initiation of insulin therapy, from the perspective of patients, were concerns about the need for frequent blood glucose checking, long-term therapy, side-effects of insulin and weight gain.

Needle phobia was found not to be among the main barriers to the initiation of insulin therapy.

The identifiable barriers did not influence the decision of the participants on whether to accept insulin therapy or not.

\section{Application to Patient Care}

It is important to address the main barriers to the initiation of insulin therapy with the patient to ensure their acceptance and adherence. Health education to diabetic patients must include clear messages on the common myths and misperceptions of insulin therapy.

Community awareness campaigns are needed to improve awareness about the safety and value of insulin therapy for patients with diabetes mellitus.

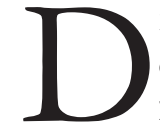

IABETES MELLITUS (DM) IS CONSIDERED A chronic disease that threatens the world. In 2019, the International Diabetes Federation reported that 463 million people worldwide had diabetes and its related deaths were estimated at 4.2 million. Unfortunately, $72 \%$ of people with diabetes are in the age range of 20-64 years, which creates a high economic burden on majority of the countries. ${ }^{1}$

Type 1 diabetes (T1DM) is usually caused by an absolute insulin deficiency that results from the destruction of beta cells of the pancreas islets. ${ }^{2}$ Type 2 diabetes (T2DM) is the most common type of diabetes and it results from a progressive insulin secretory defect, in addition to insulin resistance. ${ }^{2}$
Numerous pharmacological options are widely available for the management of diabetes. These include commonly used options such as metformin, sulphonylureas, meglitinides, thiazolidinediones and DPP-4 inhibitors. Other classes of drugs that have been recently added include GLP-1 receptor agonists and SGLT2 inhibitors. ${ }^{2}$ However, since diabetes is a progressive disease and oral hypoglycaemic agents (OHA) eventually lose their effectiveness, a big proportion of diabetic patients will eventually need to switch to insulin therapy, either alone or along with OHA. Insulin therapy is still considered the most effective pharmacological option for the treatment of diabetes. ${ }^{2}$ Moreover, studies have shown that early 
initiation of insulin therapy resulted in lower rate of complications and a slower decline in the function of beta cells of the pancreas. ${ }^{3-5}$

Poor glycaemic control is multifactorial: it could be due to healthcare workers-related factors, patient-related factors such as poor adherence, or factors related to the disease itself. ${ }^{6,7}$ Poor control leads to multiple microvascular and macrovascular complications such as end-stage kidney disease and cardiovascular disease, which would eventually cause early death. ${ }^{7,8}$ Late initiation and refusal of insulin therapy have been identified to be among the main causes of poor glycaemic control and the development of irreversible complications. ${ }^{9}$

Patient beliefs and misconceptions have been implicated in being important barriers to insulin therapy. ${ }^{7}$ Such barriers would either lead to poor adherence or refusal of insulin therapy. Needle phobia has been identified as a main barrier and has been reported to contribute significantly to the refusal of insulin therapy. ${ }^{10}$ Usually, patients prefer oral medications over needles due to its ease and convenience. Injection-based therapy is mostly feared due to the nescience on how to inject and the misconceptions including that injections must always be administered by doctors or experienced caregivers and that they are associated with complications. ${ }^{11}$

Numerous studies have been undertaken to identify the most important barriers to the initiation of insulin therapy. ${ }^{10-15}$ These barriers include criticisms such as 'insulin must be administered life-long,' 'insulin could cause organ damage' and 'insulin is a treatment option only for severe disease.12 Additionally, fear of stigma, lack of emotional support from the people around them and lack of support from their own physicians. ${ }^{13}$ Moreover, studies have revealed these barriers to be influenced by culture and healthcare system-related factors, which are unique and different for each country in the world. ${ }^{8,13,14,16}$ Besides, focused health education on insulin therapy and appropriate self-care management have been recommended as being crucial to address these barriers. ${ }^{17}$

Hence, it is important to study the barriers to insulin therapy and find appropriate strategies to address each barrier to optimise the management of diabetic patients. The lack of any previous published studies addressing this important area necessitates, with even greater importance, that this study be conducted.

Thus, this study aimed to identify the main barriers to the initiation of insulin therapy among patients with DM in Oman and estimate the influence of these barriers on the decision of patients to accept or reject insulin therapy.

\section{Methods}

This cross-sectional study was conducted at the Diabetes Clinics at Sultan Qaboos University Hospital, Bowsher Polyclinic and A'seeb Polyclinic, Muscat, Oman, from May to December 2019. All diabetic patients who were $\geq 18$ years old and who attended these clinics during the study period were invited to participate in the study, except those who were known to have severe mental disorders. A minimum sample size of 195 patients was calculated based on anecdotal evidence of an estimated barrier prevalence rate of $15 \%$ with a $5 \%$ margin of error and $95 \%$ confidence interval.

The patients, who agreed to participate, were interviewed in person by a main investigator to fill out a pre-prepared questionnaire and were given an overview about insulin therapy (indications, types, methods of initiation and administration). Convenience sampling method was used to recruit eligible participants from the three diabetes clinics. The questionnaire comprised the following items: demographic data (age, gender, occupation, monthly income and education), diabetes-related information (type of diabetes, type of treatment and number of injections per day, if on insulin therapy) and 19 specific items on barriers to insulin therapy. These items were adopted and modified from previous similar studies. ${ }^{18-20}$ The constructed questionnaire was subsequently validated by three subject experts for content, appropriateness and relevance. A four-point Likert scale was used to classify the importance of each barrier as 'not at all important', 'sometimes important', 'frequently important' or 'very important'. A three-point scale was used to determine the impact of each barrier on the patients' acceptance or rejection to insulin therapy as 'accept insulin therapy', 'borderline' or 'reject insulin therapy'. The questionnaire was translated from English to Arabic following the standard procedure of forward-and-backward translation. The questionnaire was administered in Arabic.

A pilot study involving 20 participants was carried out to test the reliability of the questionnaire, and it achieved a Cronbach's alpha of $82.1 \%$. The data were entered and analysed in both Epidata version 4.6 and using the Statistical Package for the Social Sciences (SPSS) Version 24.0 (IBM Corp., Armonk, New York, USA). Percentages and frequencies were among the descriptive statistics that were used, while continuous data were presented with mean and standard deviation. For the prevalence, 95\% confidence intervals were reported.

Informed consent was requested from all the participants prior to their enrolment in the study. The 
ethical approval for this study was granted from the Medical Research Ethics Committee (MREC) of the College of Medicine and Health Sciences of Sultan Qaboos University (MREC\#1655).

\section{Results}

In total, 218 of the 233 patients who were initially invited (response rate: 93\%) agreed to participate in the study. None were excluded based on the exclusion criteria of the study. A total of 17 questionnaires were excluded due to significant missing information; thus, 201 remaining questionnaires were eventually analysed.

The average age of the participants was $40.54 \pm$ 15.17 years. There were $122(60.7 \%)$ male participants and 91 (45.3\%) were employed while 110 (54.7\%) were retired or unemployed. A total of 88 (43.8\%) had a diploma, bachelor and higher level qualification, while 113 (56.2) had a secondary, intermediate or primary school qualifications or were illiterate. Only 14 (7.0\%) participants had a low income ( $<300$ Omani Rials) and the remaining had moderate to high income (300$\geq 1,000$ Omani Rials) [Table 1].

The majority of patients $(\mathrm{n}=84,41.8 \%)$ had T1DM, 63 (31.3\%) had T2DM and 54 (26.9\%) were unaware of the type of diabetes they had.

Data analysis revealed the following as the most common barriers: frequent blood glucose checking for 73 (36.3\%) participants, long-term insulin injections for 68 (33.8\%), side-effects of insulin for 60 (29.9\%), weight gain for 59 (29.4\%), inconvenience of the insulin injections for 58 (28.9\%), busy life schedule for 55 (27.6\%), dependence on insulin for 55 (27.4\%), hypoglycemia for 48 (24\%), pain and discomfort of injections for 46 (22.9\%) and lifestyle interference for $45(22.5 \%)$ participants. Needle phobia was considered a barrier only by a minority of the participants ( $\mathrm{n}=$ 18, 9\%; 95\% confidence interval: 5-14) [Figure 1 and Table 2].

Other barriers such as those related to difficulty to learn, difficulty to inject due to physical disabilities, lack of support from family, fear of stigma or feeling of personal failure had no major impact and were not considered barriers. All of these constituted less than 20\% [Table 2].

Overall, 125 (62.2\%) patients were willing to initiate insulin therapy despite the presence of barriers to insulin therapy and only 20 (10\%) of them were influenced by these barriers to such a degree that they had to reject insulin therapy. The remaining 56 (27.8\%) patients were still hesitant about insulin initiation and
Table 1: Socio-demographic and clinical characteristics of the study population $(\mathrm{N}=201)$

\begin{tabular}{lc} 
Variables & $\mathbf{n}(\%)$ \\
Mean age in years \pm SD & $40.54 \pm 15.17$ \\
Gender & \\
Male & $122(60.7)$ \\
Female & $79(39.3)$ \\
Occupational status & \\
Unemployed & $73(36.3)$ \\
Employed & $83(41.3)$ \\
Self-employed & $8(4.0)$ \\
Retired & $37(18.4)$ \\
Monthly income in OMR & \\
Low (<300) & $14(7.0)$ \\
Moderate (300-1000) & $108(53.7)$ \\
High (>1000) & $79(39.3)$ \\
Education & $54(15.9)$ \\
Illiterate & $56(27.9)$ \\
Primary & $54(26.9)$ \\
Intermediate & $14(7.0)$ \\
Secondary school & $13(6.5)$ \\
Diploma & $28(13.9)$ \\
Bachelor's and above & $58(28.9)$ \\
Diabetes type & $32(1.8)$ \\
Type 1 & \\
Dype 2 & \\
\hline
\end{tabular}

\section{Type of treatment}

No medication $\quad 4(2.0)$

Tablets $\quad 46(22.9)$

Insulin injections $95(47.3)$

Tablets and insulin $\quad 50(24.9)$

Insulin pump $6(3.0)$

Number of injections per day

$1 \quad 15(9.9)$

$225(16.6)$

$3 \quad 42(27.8)$

$4.64(42.4)$

$>4 \quad 5(3.3)$

$S D=$ standard deviation; $O M R=$ Omani Rial . 


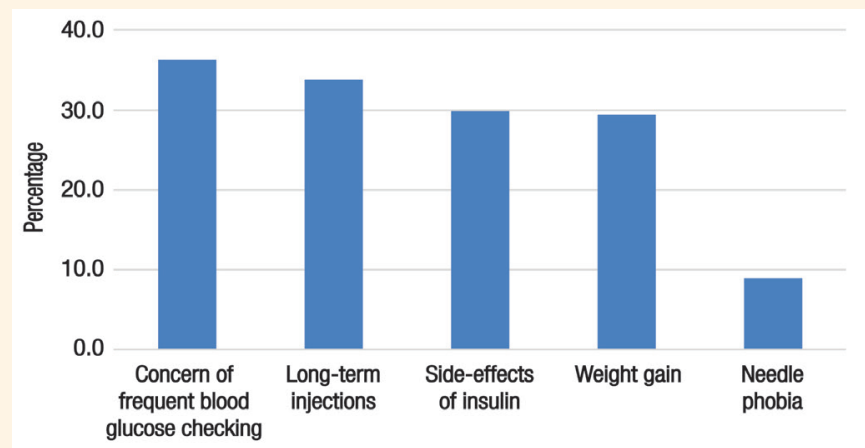

Figure 1: The most common barriers to the initiation of insulin therapy amongst the participants of the study.

Table 2: The responses of the participants regarding the importance of each barrier to initiation of insulin therapy

\begin{tabular}{|c|c|c|c|c|}
\hline \multirow[t]{2}{*}{ Items } & \multicolumn{4}{|c|}{ n (\%) } \\
\hline & $\begin{array}{l}\text { Not at all } \\
\text { important }\end{array}$ & $\begin{array}{l}\text { Sometimes } \\
\text { important }\end{array}$ & $\begin{array}{l}\text { Frequently } \\
\text { important }\end{array}$ & Very important \\
\hline Needle phobia & $146(72.6)$ & $37(18.4)$ & $10(5.0)$ & $8(4.0)$ \\
\hline Feeling of personal failure* & $14.1(70.5)$ & $39(19.5)$ & $9(4.5)$ & $11(5.5)$ \\
\hline Pain, discomfort of injections & $83(41.3)$ & $72(35.8)$ & $29(14.4)$ & $17(8.5)$ \\
\hline Lifestyle interference* & $100(50.0)$ & $55(27.5)$ & $27(13.5)$ & $18(9.0)$ \\
\hline Concern of hypoglycaemia* & $81(40.5)$ & $71(35.5)$ & $30(15.0)$ & $18(9.0)$ \\
\hline Concern of insulin side-effects & $90(44.8)$ & $51(25.4)$ & $32(15.9)$ & $28(13.9)$ \\
\hline $\begin{array}{l}\text { Concern of insulin as a cause of long-term diabetes } \\
\text { complications }\end{array}$ & $95(47.3)$ & $38(18.9)$ & $29(14.4)$ & $39(19.4)$ \\
\hline Remarkably busy life schedule* & $92(46.2)$ & $52(26.1)$ & $25(12.6)$ & $30(15.1)$ \\
\hline Concern of weight gain & $110(54.7)$ & $32(15.9)$ & $25(12.4)$ & $34(16.9)$ \\
\hline Concern of dependence on insulin & $108(53.7)$ & $38(18.9)$ & $28(13.9)$ & $27(13.4)$ \\
\hline Concern of frequent blood glucose checking & $96(47.8)$ & $32(15.9)$ & $38(18.9)$ & 35 (17.4) \\
\hline Inconvenience & $110(54.7)$ & $33(16.4)$ & $24(11.9)$ & $34(16.9)$ \\
\hline Concern of stigma* & $151(75.5)$ & $28(14.0)$ & $11(5.5)$ & $10(5.0)$ \\
\hline Difficult to learn & $164(81.6)$ & $22(10.9)$ & $4(2.0)$ & $11(5.5)$ \\
\hline Difficultly to inject due to physical disabilities & $152(75.6)$ & $22(10.9)$ & $16(8.0)$ & $11(5.5)$ \\
\hline Cannot inject myself & $171(85.1)$ & $11(5.5)$ & $4(2.0)$ & $15(7.5)$ \\
\hline No other person who can inject me & $155(77.1)$ & $20(10.0)$ & $14(7.0)$ & $12(6.0)$ \\
\hline Lack of support from family for insulin treatment & $164(81.6)$ & $15(7.5)$ & $15(7.5)$ & $7(3.5)$ \\
\hline Lack of general family support & $172(85.6)$ & $15(7.5)$ & $10(5.0)$ & $4(2.0)$ \\
\hline
\end{tabular}

"The sum of the responses of this item is $<201$ due to missing responses.

requested more time to decide [Table 3].

\section{Discussion}

This study identified the following as the main barriers to the initiation of insulin therapy: concern of frequent blood glucose checking, long-term injections, sideeffects of insulin and weight gain. Needle phobia was not found to be a major concern for the participants of this study. Furthermore, these barriers did not seem to influence the decision of the subjects as to whether or not they accept the insulin therapy.

The concern of frequent blood glucose checking was among the main barriers in this study. In this study, the two main factors that may have contributed 
Table 3: The responses of the participants regarding the impact of each barrier as to accept or reject insulin therapy

\begin{tabular}{|c|c|c|c|}
\hline \multirow[t]{2}{*}{ Items } & \multicolumn{3}{|c|}{ n (\%) } \\
\hline & $\begin{array}{l}\text { Accept insulin } \\
\text { therapy }\end{array}$ & Borderline & Reject insulin therapy \\
\hline Needle phobia & $146(72.6)$ & $47(23.4)$ & $8(4.0)$ \\
\hline Feeling of personal failure* & $141(70.5)$ & $48(24)$ & $11(5.5)$ \\
\hline Pain and discomfort of injections & $83(41.3)$ & $101(50.2)$ & $17(8.5)$ \\
\hline Lifestyle interference* & $100(50.0)$ & $82(41)$ & $18(9.0)$ \\
\hline Concern of hypoglycemia* & $81(40.5)$ & $101(50.5)$ & $18(9.0)$ \\
\hline Concern of insulin side-effects & $90(44.8)$ & $83(41.3)$ & $28(13.9)$ \\
\hline $\begin{array}{l}\text { Concern of insulin as a cause of long-term diabetes } \\
\text { complications }\end{array}$ & $95(47.3)$ & $67(33.3)$ & 39 (19.4) \\
\hline Remarkably busy life schedule* & $92(46.2)$ & $77(38.7)$ & $30(15.1)$ \\
\hline Concern of weight gain & $110(54.7)$ & $57(28.3)$ & $34(16.9)$ \\
\hline Concern of dependence on insulin & $108(53.7)$ & $66(32.8)$ & $27(13.4)$ \\
\hline Concern of frequent blood glucose checking & $96(47.8)$ & $70(34.8)$ & $35(17.4)$ \\
\hline Inconvenience & $110(54.7)$ & $57(28.3)$ & $34(16.9)$ \\
\hline Concern of stigma* & $151(75.5)$ & 39 (19.5) & $10(5.0)$ \\
\hline Difficult to learn & $164(81.6)$ & $26(12.9)$ & $11(5.5)$ \\
\hline Difficult to inject due to physical disabilities & $152(75.6)$ & $38(18.9)$ & $11(5.5)$ \\
\hline Cannot inject myself & $171(85.1)$ & $15(7.5)$ & $15(7.5)$ \\
\hline No other person that can inject me & $155(77.1)$ & $34(17)$ & $12(6.0)$ \\
\hline Lack of support from family for insulin treatment & 164. (81.6) & $30(15)$ & $7(3.5)$ \\
\hline Lack of general family support & $172(85.6)$ & $25(12.5)$ & $4(2.0)$ \\
\hline Overall & $125(62.2)$ & $56(27.8)$ & $20(10)$ \\
\hline
\end{tabular}

*The sum of the responses of this item is $<201$ due to missing responses.

to this finding were the low level of education among the participants and the fact that the majority of them were on insulin therapy, which usually requires frequent blood glucose monitoring. Wallace and Matthews attributed this barrier either to the fear of hypoglycaemia owing to patient beliefs that insulin has an immediate action or to the patient's worry about hyperglycaemia and their intention to seek rapid improvement. ${ }^{7}$

Other major barriers identified in this study included concern of long-term injections and its sideeffects. Similar findings were demonstrated in a study that was conducted in the USA. Insulin therapy was feared, as the subjects in the study mistakenly believed that insulin medication could lead to amputations (15\%), renal failure (32\%), strokes (19\%), heart attacks, blindness (20\%) and early death (19\%). ${ }^{10}$ Another study demonstrated the concern of hypoglycaemia as a barrier to insulin initiation, especially among those who had increased their doses of insulin. ${ }^{11}$
Most patients with T2DM are overweight at the onset of diagnosis with an average body mass index of $27.5 \mathrm{~kg} / \mathrm{m}^{2}$ as shown in the United Kingdom Prospective Diabetes Study. ${ }^{22}$ The presence of two medical problems, namely diabetes and obesity, at an early stage, discourages the patients and physicians to adopt any treatment that might induce weight gain. Indeed, studies have revealed fear of weight gain as a big concern in such patients, especially if they have been treated with insulin. ${ }^{7,11}$

Usually, people prefer oral drugs over needles due to the pain and discomfort of injections. ${ }^{9,14,15}$ In the current study, 46 (22.9\%) patients found insulin treatment inconvenient due to this reason.

Most people think that insulin restricts their life schedule and themselves from their work or sports. ${ }^{7,21}$ Furthermore, it might be associated with carrying the needles wherever the patients go unlike oral medications, which are easier to carry especially for frequent travellers. ${ }^{9,15}$ These findings are consistent 
with the results of the current study, which showed that insulin initiation was rejected due to lifestyle interference (22.5\%), inconvenience of the insulin needles treatment $(28.9 \%)$ and busy life schedule (27.6\%).

Insulin therapy might be initiated for a short period to combat severe hyperglycaemia such as in newly diagnosed T2DM patients. However, some of these patients might be reluctant to start insulin due to their fear of dependence to insulin and the concern of being dependent on it life-long. ${ }^{9,10,17}$ This fact was also demonstrated in $55(27.3 \%)$ patients.

Difficulty to learn is an infrequent barrier to insulin initiation that was documented in 15 (7.5\%) patients of this study. This can be seen more commonly among inhabitants of rural areas where people are expected to not have enough education and access to medical services. Thus, their knowledge about the disease and available therapies might be lacking. ${ }^{21}$ Moreover, few patients have concern of stigma, which is mostly related to the use of insulin, administration of insulin injection in public or the shame of telling people of their chronic disease, and believe that it might affect their relationships or connections with others. $^{12}$

The prevalence of the needle phobia in the current study was only 9\%, as opposed to the findings of a qualitative study, which was conducted in five different countries (Germany, Sweden, The Netherlands, United Kingdom and USA). The researchers reported a high prevalence (43.7\%) of needle phobia among their subjects. ${ }^{17}$ This phobia seemed to be unaffected by the variation in who gives the injection or where it is given. ${ }^{17}$ A systematic review conducted by McLenon et al. reported a higher prevalence of needle phobia in females, young adults and in specific countries. ${ }^{23}$ Thus, this low prevalence in the present study could be attributed to the predominant age and gender of the participants, in addition to country-specific factors.

Most participants in this study were willing to initiate insulin therapy despite confirming the presence of barriers. This might be explained by the possibility that these barriers are not strong enough to influence their final decision on insulin therapy. Moreover, concerns about the complications of poorly controlled diabetes and side-effects of other treatments could also lead to insulin acceptance. ${ }^{12}$

The findings of this study are important since it gives physicians an insight about the barriers to insulin initiation from the perspective of the patients. This is the first critical step to develop strategies that appropriately address these myths and misconceptions to aim for the better care of diabetic patients in Oman. ${ }^{16,24,25}$
This study has several important limitations. The questionnaire-based design of the study is dependent on self-reported responses, which are usually influenced by social desirability. Since most responses obtained from the interviews, particularly those related to the barriers among participants who were already on insulin therapy, are dependent on their ability for recall, recall information bias can be considered a limitation. The responses to questionnaires are usually influenced by the level of education and there was a big proportion of subjects who had low level of education. Moreover, this tool is limited by its ability to capture attitudes and not real behaviours. The relationship between barriers and the decision to accept or reject insulin is also hypothetical and the responses might not reflect the final decision. The study population, which is limited to the attendants of outpatient clinics, is not representative of the general population, which affects the generalisability of the findings.

\section{Conclusion}

This study addressed the barriers that might influence the decision to accept or reject insulin therapy amongst diabetic patients in Oman. The main barriers were the concern of frequent blood glucose checking, longterm therapy and side-effects. Most study participants reported no impact of these barriers on their final decision to accept or reject insulin therapy. Contrary to the findings of other similar studies, needle phobia was found not to be a major barrier to insulin initiation in this study.

\section{AUTHORS' CONTRIBUTION}

AAF, MA, SAQ, SAG, SP and AAM conceptualised and designed the study. AAF, MA, AAK, SAQ and SAG collected the data. AAF, SP and AAM analysed and interpreted the data. AAF, MA, SAQ and AAM drafted the manuscript. All authors approved the final version of the manuscript.

\section{CONFLICT OF INTEREST}

The authors declare no conflicts of interest.

\section{FUNDING}

No funding was received for this study.

\section{References}

1. International Diabetes Federation. IDF Diabetes Atlas 9th edition 2019. https://www.diabetesatlas.org Accessed: May 2021.

2. Classification and Diagnosis of Diabetes: Standards of Medical Care in Diabetes-2018. Diabetes Care 2018; 41:S13-27. https:// doi.org/10.2337/dc18-S002. 
3. Li Y, Xu W, Liao Z, Yao B, Chen X, Huang Z, et al. Induction of long-term glycemic control in newly diagnosed type 2 diabetic patients is associated with improvement of $\beta$-cell function. Diabetes Care 2004; 27:2597-602. https://doi.org/10.2337/dia care.27.11.2597.

4. Weng J, Li Y, Xu W, Shi L, Zhang Q, Zhu D, et al. Effect of intensive insulin therapy on beta-cell function and glycaemic control in patients with newly diagnosed type 2 diabetes: a multicentre randomised parallel-group trial. Lancet 2008; 371:1753-60. https://doi.org/10.1016/S0140-6736(08)60762-X.

5. Harrison LB, Adams-Huet B, Raskin P, Lingvay I. $\beta$-cell function preservation after 3.5 years of intensive diabetes therapy. Diabetes Care 2012; 35:1406-12. https://doi.org/10.2337/dc11-2170.

6. Bin Rsheed A, Chenoweth I. Barriers that practitioners face when initiating insulin therapy in general practice settings and how they can be overcome. World J Diabetes 2017; 8:28-39. https://doi.org/10.4239/wjd.v8.i1.28.

7. Wallace TM, Matthews DR. Poor glycaemic control in type 2 diabetes: a conspiracy of disease, suboptimal therapy and attitude. QJM 2000; 93:369-74. https://doi.org/10.1093/qjmed/93.6.369.

8. Alramadan MJ, Afroz A, Hussain SM, Batais MA, Almigbal TH, Al-Humrani HA, et al. Patient-related determinants of glycaemic control in people with type 2 diabetes in the gulf cooperation council countries: A systematic review. J Diabetes Res 2018; 9389265. https://doi.org/10.1155/2018/9389265.

9. Polonsky WH, Fisher L, Guzman S, Villa-Caballero L, Edelman SV Psychological insulin resistance in patients with type 2 diabetes: The scope of the problem. Diabetes Care 2005; 28: 2543-5. https://doi.org/10.2337/diacare.28.10.2543.

10. Karter AJ, Subramanian U, Saha C, Crosson JC, Parker MM, Swain BE, et al. Barriers to insulin initiation. Diabetes Care 2010; 33:733-5. https://doi.org/10.2337/dc09-1184.

11. Petznick AM. Identifying and addressing barriers to insulin acceptance and adherence in patients with type 2 diabetes mellitus. J Am Osteopath Assoc 2013; 113:S6-16.

12. Abu Hassan $\mathrm{H}$, Tohid $\mathrm{H}$, Amin MR, Long Bidin MB, Muthupalaniappen L, Omar K. Factors influencing insulin acceptance among type 2 diabetes mellitus patients in a primary care clinic: A qualitative exploration. BMC Fam Prac 2013; 14:164. https://doi.org/10.1186/1471-2296-14-164.

13. Tan AM, Muthusamy L, Ng CC, Phoon KY, Ow JH, Tan NC. Initiation of insulin for type 2 diabetes mellitus patients: what are the issues? A qualitative study. Singapore Med J 2011; $52: 801-9$

14. Chen K, Tseng H, Huang Y, Chuang Y. The barriers to initiating insulin therapy among people with type 2 diabetes in taiwan A qualitative study. J Diabetes Metab 2012; 3:3-6. https://doi. org/10.4172/2155-6156.1000194.
15. Wibisono AH, Lestari A, Sorensen L, Hill P. Fear of injections among people with type 2 diabetes: Overview of the problem. J Diabetes Nurs 2017; 21:91-5

16. Peyrot M, Rubin RR, Lauritzen T, Skovlund SE, Snoek FJ, Matthews DR, et al. International DAWN Advisory Panel. Resistance to insulin therapy among patients and providers: Results of the cross-national Diabetes Attitudes, Wishes, and Needs (DAWN) study. Diabetes Care 2005; 28:2673-9. https:// doi.org/10.2337/diacare.28.11.2673.

17. Brod M, Alolga SL, Meneghini L. Barriers to initiating insulin in type 2 diabetes patients: Development of a new patient education tool to address myths, misconceptions and clinical pealities. Patient 2014; 7:437-50. https://doi.org/10.1007/s40271-014-0068-x.

18. Hussein A, Mostafa A, Areej A, Mona AM, Shimaa A, Najd AG, et al. The perceived barriers to insulin therapy among type 2 diabetic patients. Afr Health Sci 2019; 19:1638-46. https://doi. org/10.4314/ahs.v19i1.39.

19. Shafie Pour MR, Sadeghiyeh T, Hadavi M, Besharati M, Bidaki R. The barriers against initiating insulin therapy among patients with diabetes living in Yazd, Iran. Diabetes Metab Syndr Obes 2019; 12:1349-54. https://doi.org/10.2147/DMSO.S200867.

20. Russell-Jones D, Pouwer F, Khunti K. Identification of barriers to insulin therapy and approaches to overcoming them. Diabetes Obes Metab 2018; 20:488-96. https://doi.org/10.1111/dom.13132.

21. Tan WL, Asahar SF, Harun NL. Insulin therapy refusal among type II diabetes mellitus patients in Kubang Pasu district, Kedah, Malaysia. Singapore Med J 2015; 56:224-7. https://doi.org/10.11 622/smedj.2014170.

22. King P, Peacock I, Donnelly R. The UK prospective diabetes study (UKPDS): Clinical and therapeutic implications for type 2 diabetes. Br J Clin Pharmacol 1999; 48:643-8. https://doi.org/10.10 46/j.1365-2125.1999.00092.x

23. McLenon J, Rogers MAM. The fear of needles: A systematic review and meta-analysis. J Adv Nurs 2019; 75:30-42. https:// doi.org/10.1111/jan.13818.

24. Balogh EG, Perez-Nieves M, Cao D, Hadjiyianni II, Ashraf N, Desai U, et al. Key strategies for overcoming psychological insulin resistance in adults with type 2 diabetes: The UK subgroup in the EMOTION Study. Diabetes Ther 2020; 11:1735-44. https:// doi.org/10.1007/s13300-020-00856-4.

25. Peyrot M, Rubin RR, Kruger DF, Travis LB. Correlates of insulin injection omission. Diabetes Care 2010; 33:240-5. https://doi. org/10.2337/dc09-1348. 